\title{
Análise do desempenho em atletas de elite no "Ironman" Brasil entre os anos de 2003 a 2010
}

\author{
CDD. 20.ed. 796.073 \\ 796.426 \\ 796.6 \\ 797.2
}

\author{
Everton Crivoi CARMO*/** \\ Henrique Afonso RAMOS* \\ Licia ELIAS" \\ Victor Tavares ALVES" \\ Carlos UGRINOWITSCH ${ }^{*}$ \\ Valmor Alberto Augusto TRICOLI" \\ Hamilton ROSCHEL*
}

*Escola de Educação Física e Esporte,

Universidade de São Paulo.

${ }^{* *}$ Centro Universitário SENAC - Santo Amaro.

\section{Resumo}

0 objetivo do presente estudo foi analisar a evolução no desempenho no "Ironman" Brasil entre os anos de 2003 a 2010. Adicionalmente, verificamos qual modalidade apresentou maior influência sobre o desempenho final na prova. Foram analisados os 10 primeiros colocados da categoria elite masculino de cada ano avaliado. As diferenças entre os tempos foram analisadas por ANOVA, seguida por "post-hoc" de Tukey. 0 coeficiente de correlação de Pearson foi empregado para investigar a associação entre 0 desempenho total e o desempenho nas diferentes modalidades isoladas. Os resultados mostraram não ter havido evolução no desempenho ao longo dos anos, sendo observada grande variação nos resultados. Foi observada, ainda, correlação entre o tempo de prova no ciclismo e o tempo total $(r=0,82 ; p<0,01)$, sendo que o atleta com melhor tempo no ciclismo foi, também, o campeão da prova em $75 \%$ das vezes. Por fim, concluímos que não houve evolução no desempenho ao longo dos anos analisados. Adicionalmente, o ciclismo parece ser a modalidade determinante para o sucesso do atleta no "Ironman" Brasil.

Palavras-chave: Desempenho esportivo; Corrida; Ciclismo; Natação.

\section{Introdução}

Durante uma prova de triátlon os atletas devem realizar sequencialmente três diferentes modalidades de resistência: a natação, o ciclismo e a corrida ${ }^{1}$. Dentre as suas diferentes categorias, o triátlon de longa duração talvez seja uma das mais tradicionais da modalidade e uma das provas de resistência mais desafiadoras do mundo e, por esse motivo, recebe o nome de "Ironman". Nesta, os atletas devem realizar 3,8 km de natação, $180 \mathrm{~km}$ de ciclismo e $42 \mathrm{~km}$ de corrida ${ }^{1,2}$.

As características fisiológicas do triátlon são similares às demandas das modalidades esportivas analisadas individualmente. No entanto, as demandas fisiológicas durante a prova são bem mais complexas do que nas provas simples uma vez que, se a intensidade do esforço for muito alta em uma das modalidades, ela poderá comprometer o rendimento nas modalidades seguintes ${ }^{3}$. Sendo o triátlon de longa distância considerado uma prova de "ultra-endurance", ela é realizada em intensidades próximas ao limiar anaeróbio ${ }^{4,5}$.

A primeira prova oficial de "Ironman" foi realizada em 1978, em Honolulu, Havaí1. Já no Brasil, o "Ironman" foi realizado pela primeira vez em 1982, na cidade de Porto Seguro, Bahia, sem caráter competitivo e com percurso inferior ao tradicional. A partir de 2001 a prova foi transferida para a cidade de Florianópolis, Santa Catarina, apresentando um rápido crescimento no número de participantes. Atualmente, quase 2000 atletas competem nesta que é considerada uma das mais importantes provas da modalidade, já que é classificatória para a etapa do Havaí.

Com a popularização do triátlon "Ironman" e o crescente número de competidores nas diferentes etapas do circuito mundial, o nível profissional dos 
atletas aumentou ao longo dos anos ${ }^{1,7-9}$. Associado a isso, novos equipamentos foram desenvolvidos ("aerobars", quadros de carbono e potenciômetros) e novas metodologias de treinamento têm sido propostas (inclusão do treinamento intervalado de alta intensidade, do treinamento de força el ou potência e a utilização de estratégias como o "taper") os quais vem contribuindo para a melhora do desempenho esportivo ${ }^{2,5-9}$. No entanto, poucos estudos analisaram a evolução do desempenho em provas de "Ironman".

Em um estudo retrospectivo sobre o desempenho na prova do Havaí entre os anos de 1987 a 2007, observou-se uma melhora expressiva dos tempos entre os 10 melhores colocados durante os anos 80. Interessantemente, nas duas décadas seguintes, a melhora foi bem menos acentuada ${ }^{1}$. Apesar do "Ironman" Brasil fazer parte do circuito mundial e ser uma prova seletiva para a etapa no Havaí, não

\section{Método}

Foram analisados os resultados obtidos no "Ironman" Brasil pela categoria Elite Masculina entre os anos de 2003 a 2010. Os dados foram adquiridos por meio do site www.ironmanbrasil.com.br e a sua utilização, sem a divulgação de nomes, foi autorizada pela empresa Latin Sports SA, responsável pela organizaçáo da prova e detentora dos diretos da marca "Ironman" Brasil.

Embora o primeiro "Ironman" Brasil tenha sido realizado em 1982, foram analisados apenas os resultados a partir de 2003, ano em que os tempos de prova passaram a ser aferidos com maior precisão e confiabilidade. Foram analisados os tempos totais de prova e os tempos parciais para cada uma das modalidades disputadas: natação, ciclismo e corrida dos 10 melhores colocados da categoria.

\section{Resultados}

O desempenho dos 10 primeiros colocados na categoria elite masculina no tempo total de prova e no tempo das três modalidades analisadas isoladamente não apresentou evolução ao longo dos oito anos analisados. Houve, no entanto, grande existem, até o presente momento, análises adequadas sobre a evolução no desempenho, tanto geral quanto específico para cada modalidade, ao longo dos anos de realização da prova.

Com a evolução do conhecimento científico aplicado aos métodos de treinamento e o desenvolvimento tecnológico de novos equipamentos, esperamos observar uma melhora significante de desempenho no "Ironman" Brasil ao longo dos anos analisados. Assim, o presente estudo teve como objetivo analisar as possíveis alterações de desempenho entre os atletas da elite masculina no "Ironman" Brasil entre os anos de 2003 a 2010. Adicionalmente, foi também objetivo do estudo, verificar qual das modalidades realizadas ao longo da prova pode apresentar maior influência sobre o desempenho final do atleta, auxiliando atletas e treinadores nos seus treinamentos e na elaboração de estratégias para a melhora do desempenho.

\section{Análise estatística}

Os dados são reportados como média \pm desvio padrão (DP). Os tempos de cada ano foram tratados como observaçóes independentes. Para analisar as diferenças entre os anos foi utilizada a Anova "oneway". O "post-hoc" com ajustamento de Tukey foi utilizado em caso de $\mathrm{F}$ significante. $\mathrm{O}$ coeficiente de correlação de Pearson foi empregado para análise do nível de associação entre o desempenho total e o desempenho nas diferentes modalidades disputadas. O nível de significância adotado foi de $5 \%(\mathrm{p}<0,05)$. Todas as análises foram desenvolvidas utilizando o "software" SPSS (versão 13.0, Chicago, EUA).

variação de desempenho, conforme demonstrado na FIGURA 1.

Os valores médios dos 10 primeiros colocados no tempo total de prova e no tempo nas três diferentes modalidades de cada ano sáo descritos na TABELA 1. 

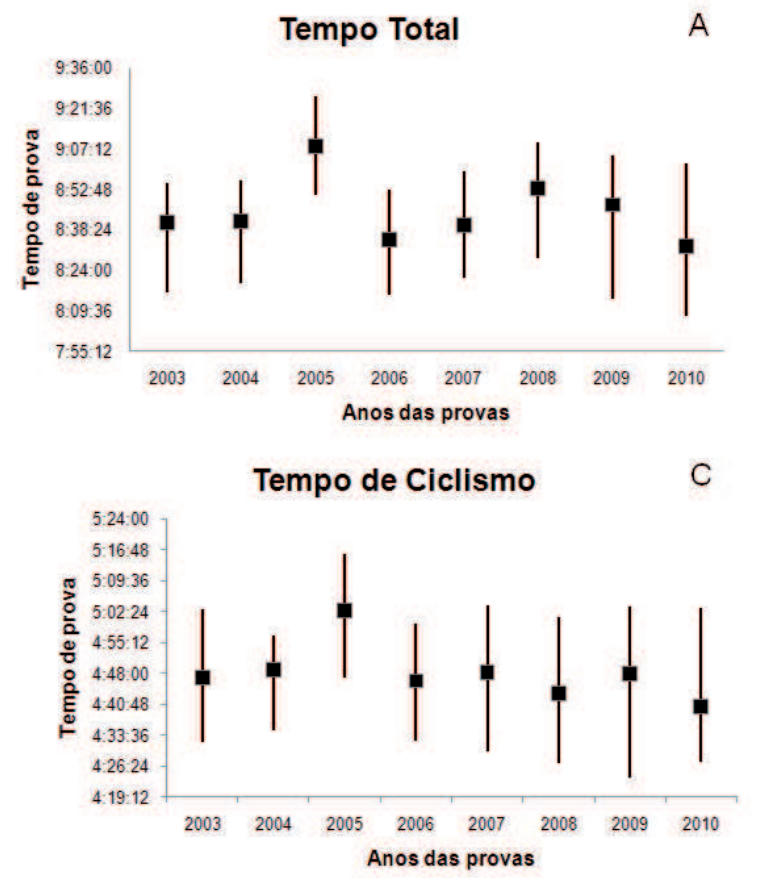

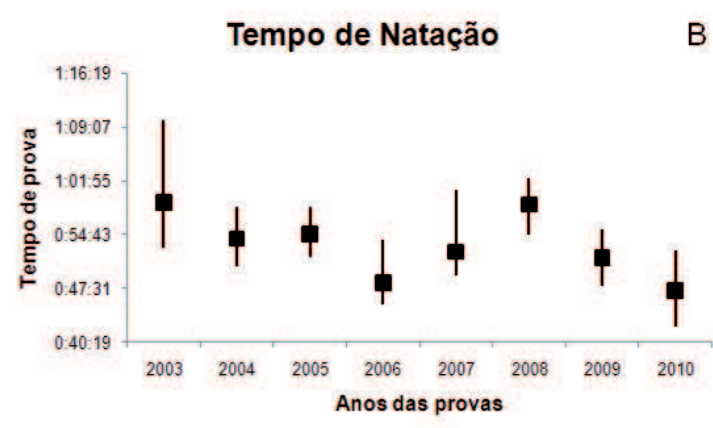

Tempo de Corrida

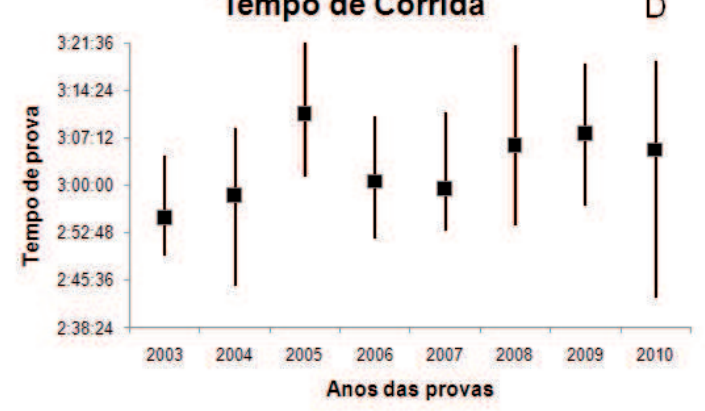

Resultados apresentados como média e minimo/máximo ao longo dos oito anos analisados no tempo total de prova e nas três diferentes modalidades isoladamente.

A: tempo total de prova; B: tempo de natação; C: tempo de ciclismo; e D: tempo de corrida.

FIGURA 1 - Representação gráfica da evolução ao longo dos anos no tempo de prova e no tempo das três diferentes modalidades isoladamente

TABELA 1 - Tempo de prova total e nas três diferentes modalidades isoladamente ao longo dos anos.

\begin{tabular}{ccccc}
\hline \multirow{2}{*}{ Anos } & \multicolumn{4}{c}{ Tempo de prova (minutos) } \\
\cline { 2 - 5 } & Total & Nataçáo & Ciclismo & Corrida \\
\hline 2003 & $520,96 \pm 15,9$ & $58,98 \pm 5,26 \ddagger$ & $286,92 \pm 11,33$ & $175,08 \pm 4,49 €$ \\
2004 & $521,51 \pm 11,27$ & $54,16 \pm 2,87$ & $288,78 \pm 7,93$ & $178,55 \pm 7,02$ \\
2005 & $548,24 \pm 10,67^{*}$ & $54,78 \pm 2,46$ & $302,58 \pm 8,92 \$$ & $190,87 \pm 7,47 \&$ \\
2006 & $514,94 \pm 12,48$ & $48,28 \pm 2,51$ & $286,07 \pm 9,28$ & $180,58 \pm 5,45$ \\
2007 & $519,89 \pm 12,41$ & $52,39 \pm 3,23$ & $288,00 \pm 10,75$ & $179,51 \pm 6,07$ \\
2008 & $533 \pm 12,88$ & $58,69 \pm 2,22 \ddagger$ & $283,12 \pm 12,06$ & $186,08 \pm 9,38$ \\
2009 & $527,24 \pm 16,93$ & $51,65 \pm 2,57$ & $287,74 \pm 13,22$ & $187,87 \pm 7,04$ \\
2010 & $512,37 \pm 17,62 \#$ & $47,19 \pm 2,73 \dagger$ & $280,05 \pm 12,00$ & $185,34 \pm 9,95$ \\
\hline
\end{tabular}

O pior desempenho no tempo total de prova ocorreu no ano de 2005 ( $\mathrm{p}<0,05$ vs. outros anos) e o melhor no ano de 2010, contudo, este foi estatisticamente diferente apenas quando comparado aos dois piores anos (2005 e 2008; $\mathrm{p}<0,05$ ).

Os piores desempenhos na nataçáo ocorreram nos anos de 2003 e 2008 ( $\mathrm{p}<0,05)$ e o melhor no ano de 2010 ( $\mathrm{p}<0,05)$. Na prova de ciclismo, o menor tempo foi observado em 2010, entretanto, não houve diferenças estátisticas em relação aos outros anos. O pior desempenho no ciclismo foi encontrado em 2005, sendo esse estatisticamente diferente dos anos de 2003, 2006, 2008 e 2010 (p
$<0,05)$. Por fim, os resultados da corrida mostram que o pior desempenho ocorreu em 2005 ( $p<0,05$ vs. 2003, 2004, 2006 e 2007) e o melhor em 2003, estatisticamente diferente dos anos de 2005, 2008, 2009 e 2010 ( $\mathrm{p}<0,05)$.

Quando os oito anos de prova foram analisados conjuntamente, foi encontrada correlação moderada entre o tempo de prova no ciclismo e o tempo total de prova $(r=0,82 ; p=0,01)$. Por outro lado, uma baixa correlação foi observada entre o tempo de natação $(\mathrm{r}$ $=0,40 ; \mathrm{p}<0,05)$ e o tempo de corrida $(\mathrm{r}=0,52 ; \mathrm{p}$ $<0,01)$ com o tempo total de prova. Os resultados das correlaçốes podem ser observados na FIGURA 2.
Os tempos são apresentados como média $\pm \mathrm{DP}$; * diferença significante em relação aos outros anos no tempo total de prova; \# diferença significante em relação aos anos de 2005 e 2008 no tempo total de prova;

† diferença significante em relação aos anos de 2003, 2004, 2005, 2007, 2008 e 2009 no tempo da natação;

$\ddagger$ diferença significante em relação aos anos de 2004 , 2006, 2007, 2009 e 2010 no tempo da natação; \$ diferença significante em relação aos anos de 2003, 2006, 2008 e 2010 no tempo de ciclismo; \& diferença significante em relação aos anos de 2003, 2004, 2006 e 2007 no tempo de corrida;

$€$ diferença significante em relação aos anos de 2005, 2008, 2009 e 2010 no tempo de corrida. 
As correlaçóes ano a ano entre o tempo total de prova e as diferentes modalidades são demonstradas na TABELA 2. Assim como observado quando todos os anos foram analisados conjuntamente, o ciclismo apresentou maiores valores de correlação com o tempo total de prova em todos os anos analisados.

A: Correlação entre o tempo de natação e o tempo total de prova; B: Correlação entre 0 tempo de ciclismo e o tempo total de prova; C: Correlação entre 0 tempo de ciclismo e o tempo total de prova.

Para a análise dos dados os resultados de todos os anos foram utilizados.
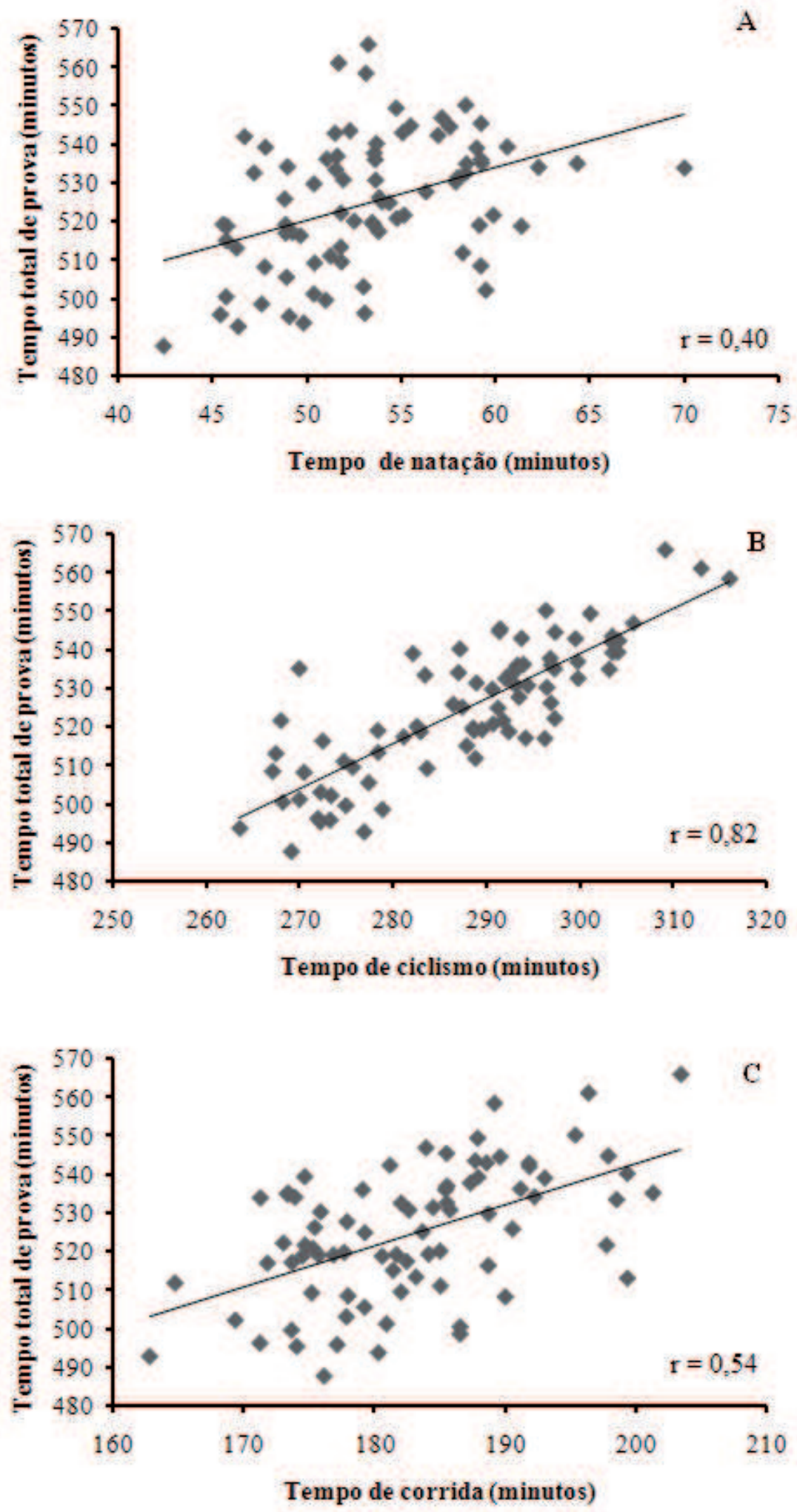

FIGURA 2 - Correlação entre o tempo total de prova e o tempo nas três diferentes modalidades.

60 • Rev Bras Educ Fís Esporte, (São Paulo) 2014 Jan-Mar; 28(1):57-64 
TABELA 2 - Correlações entre modalidades e tempo total de prova ano a ano.

\begin{tabular}{cccc}
\hline \multirow{2}{*}{ Anos } & \multicolumn{3}{c}{ Correlaçóes entre modalidades e tempo total de prova $(\mathbf{r})$} \\
\cline { 2 - 4 } & Natação & Ciclismo & Corrida \\
\hline 2003 & 0,57 & 0,95 & 0,28 \\
2004 & 0,26 & 0,89 & 0,53 \\
2006 & 0,26 & 0,75 & 0,62 \\
2007 & 0,24 & 0,87 & 0,70 \\
2008 & 0,58 & 0,87 & 0,17 \\
2009 & 0,46 & 0,81 & 0,26 \\
2010 & 0,35 & 0,90 & 0,58 \\
\hline
\end{tabular}

\section{Discussão}

Os principais resultados do nosso estudo mostram que não houve evolução no tempo do "Ironman" Brasil entre os anos de 2003 a 2010, sendo observada grande variação nos resultados ao longo dos anos. Adicionalmente, verificamos que no "Ironman" Brasil o ciclismo parece ser uma modalidade determinante para o sucesso do atleta, uma vez que os atletas mais rápidos no ciclismo foram os campeóes da prova em seis dos oito anos analisados.

No presente estudo foram avaliados os 10 primeiros colocados na categoria elite masculina. A escolha apenas dos primeiros colocados permite resultados mais precisos sobre as mudanças de desempenho ao longo dos anos, uma vez que os demais colocados apresentam resultados muito diferentes do primeiro lugar ${ }^{1}$.

O crescimento do triátlon de longa distância, o maior nível dos atletas e, consequentemente, a maior competitividade, impulsionou a realização de estudos e o melhor entendimento sobre a modalidade ao longo dos anos ${ }^{2,8-13}$. Novos conhecimentos sobre o treinamento realizado sugerem que os atletas com melhor desempenho são os que realizam maiores distâncias semanais e ritmos mais intensos ${ }^{3,14-16}$. Treinamentos específicos, priorizando variáveis como a economia de movimento, também têm sido sugeridos ${ }^{17}$. A economia de movimento parece ser determinante para o sucesso do atleta, uma vez que a melhor economia de movimento na natação permite ao atleta realizar as duas atividades subsequentes com melhor desempenho ${ }^{18}$.

Adicionalmente, avanços sobre o estudo da estratégia de corrida utilizada ${ }^{7,19}$ e o desenvolvimento de estratégias nutricionais também tem sido observados ${ }^{8,11,20}$. Assim, com o novo conhecimento adquirido sobre o triátlon de longa distância nos últimos anos, podemos supor que o desempenho dos atletas tenha melhorado. No entanto, esse fenômeno tem sido pouco avaliado.

LePERs $^{1}$ analisou a evoluçáo do desempenho no "Ironman" Havaí entre os anos 1987 a 2007. Foi observado melhora nos tempos de prova apenas nos anos 80 , sendo que após esse período, nas duas décadas seguintes, o desempenho dos atletas parece ter estabilizado. Os resultados do nosso estudo corroboram com os observados por LePERs ${ }^{1}$. Diferente do esperado, não foi observada melhora no desempenho do "Ironman" Brasil, tanto no tempo total de prova quanto no tempo das diferentes modalidades entre os anos de 2003 a 2010, sendo encontrada grande variação nos resultados.

Uma vez que o aumento de informaçáo e o desenvolvimento tecnológico dos equipamentos têm influência direta sobre o desempenho dos atletas, esperávamos observar uma redução significante no tempo de prova ao longo dos anos, principalmente no ciclismo, em que grandes mudanças tecnológicas vêm sendo realizadas desde os anos $80^{9,10}$. No entanto, a falta de melhora de desempenho no presente estudo náo pode ser atribuída à falta de evolução tecnológica ou a uma possível estabilizaçáo na preparação física dos atletas, mas sim a grande variação de desempenho ao longo dos anos, o que prejudicou a análise dos resultados.

A grande variação nos resultados ao longo dos anos pode ter sido induzida por outros fatores intervenientes sobre o desempenho, entre os quais podemos destacar as diferentes condições climáticas 
encontradas pelos atletas. Apesar deste aspecto representar uma limitação importante do estudo, as alterações climáticas são parte inerente à competição nesta modalidade, sendo este um fator de difícil controle experimental.

A temperatura da água, as mudanças de maré e as diferentes ondulações podem explicar os diferentes resultados observados na natação ${ }^{12}$. Outros fatores como a direçáo e velocidade do vento, a temperatura e umidade relativa do ar também podem explicar a variação observada no ciclismo e na corrida ${ }^{1}$.

O pior desempenho no tempo total de prova foi observado em 2005. Segundo relatos de atletas e da organização da prova, dados confirmados por meio do website www.wundergroung.com/history, no dia da prova, 29 de maio de 2005, o dia estava claro e limpo, sem nebulosidade e com temperatura média de $22{ }^{\circ} \mathrm{C}$ e ventos moderados chegando a $22 \mathrm{~km} / \mathrm{h}$. Apesar de moderado, o vento contra no percurso de ciclismo aumentou a resistência do ar, o que dificulta o seu deslocamento, aumentando o desgaste sobre o atleta e tempo gasto nesta etapa de prova. Adicionalmente, o vento de $22 \mathrm{~km} / \mathrm{h}$ pode deixar o mar forte com ondulaçóes de até um metro, com "carneiros" frequentes, o que pode explicar os altos tempos na natação. Comportamento semelhante foi observado em 2008, em que ventos de $20 \mathrm{~km} / \mathrm{h}$ e um mar com muita ondulação podem ter contribuído para o maior tempo final de prova.

Por outro lado, o melhor desempenho foi observado em 2010. Nesse ano, o dia da prova estava com o vento pela manhã com velocidade em torno de 13 $\mathrm{km} / \mathrm{h}$ e o mar estava sem correnteza, facilitando a navegaçáo e o deslocamento do atleta na água. Nesse mesmo ano foi observado o melhor desempenho no ciclismo, que pode ter sido favorecido pelo menor desgaste dos atletas durante a etapa de natação.

Como podemos observar, as mudanças climáticas podem ter influenciado diretamente os resultados, o que justifica a grande variação observada ao longo dos anos e dificulta uma análise mais precisa sobre a evolução de desempenho no "Ironman" Brasil. A variação climática também tem sido apontada por influenciar a análise da evolução do desempenho no "Ironman" Havaí1. Como exemplos, podemos citar o ano de 2004 em que o vento forte pode estar relacionado ao pior desempenho no ciclismo ou ainda as condições climáticas favoráveis nos anos de 2005 e 2006 onde os melhores desempenhos foram observados no ciclismo ${ }^{1}$. Assim, as condiçóes climáticas devem ser levadas em consideração por influenciar diretamente o desempenho do atleta.
A variação de percurso ao longo dos anos é outro fator que pode ter influenciado diretamente os resultados de desempenho e, portanto, deve ser levada em consideração. No ano de 2005 ocorreram mudanças significativas no percurso da prova. A natação deixou de ser realizada em traçado triangular, e o trajeto passou a ter formato em " $\mathrm{M}$ ", com voltas que não adentram tanto ao mar, formato utilizado até hoje. $\mathrm{Na}$ corrida, alterou-se o percurso de uma volta única de $42 \mathrm{~km}$, para três voltas de menor distância, sendo a primeira de $21 \mathrm{~km}$ e as outras duas de $10,5 \mathrm{~km}$. Essas alteraçóes podem estar relacionadas ao pior desempenho nos anos de 2005.

No triátlon de longa distância como o "Ironman", a natação representa, em média, $11 \%$, o ciclismo $52 \%$ e a corrida $35 \%$ do tempo total de prova. O esforço realizado em uma modalidade pode influenciar diretamente sobre as outras e sobre o desempenho total ${ }^{3,7}$. Assim, entender qual a modalidade é mais decisiva para o desempenho final na prova e traçar a melhor estratégia parece ser fator determinante para o sucesso do atleta. No "Ironman" Brasil, a modalidade que mais está associada ao desempenho total de prova é o ciclismo. Foi observada uma correlação moderada entre o tempo de prova no ciclismo e o tempo total, sendo que o atleta que fez o melhor tempo no ciclismo foi, também, o campeão da prova em seis dos oito anos analisados ( $75 \%$ das vezes). Por outro lado, a nataçáo foi a que apresentou menor correlaçáo. $\mathrm{O}$ ciclismo pode ter grande importância para o desempenho total por ser a modalidade mais longa da prova, tomando sempre mais da metade do tempo total ${ }^{1,7}$. Assim, um desempenho ruim no ciclismo dificilmente poderá ser compensado durante a corrida.

Os resultados do presente estudo podem ser levados em consideração por técnicos e atletas para o planejamento mais adequado do treinamento para a etapa do "Ironman" Brasil. Uma vez que o ciclismo parece ser a modalidade determinante do desempenho, o treinamento desta modalidade poderia ser priorizado, utilizando maiores distâncias e treinos mais intensos ${ }^{3,14,16}$.

Apesar da natação não apresentar alta correlação com o desempenho no "Ironman" Brasil, pode influenciar diretamente a etapa de ciclismo e, consequentemente, o desempenho final. Assim, o treinamento de natação também deve ser bem planejado para que o atleta termine a etapa bem posicionado, mas sem um desgaste físico excessivo. Neste sentido, treinadores poderiam trabalhar estratégias de treinamento visando à economia de 
movimento do atleta ${ }^{18}$. Outro ponto importante que deve ser levado em consideração é a utilização de bicicletas específicas para provas de longas distâncias, com angulação adequada do canote em relação ao quadro, o que permite ao atleta poupar a musculatura utilizada durante a corrida.

A estratégia de prova utilizada também deve ser levada em consideração ${ }^{7,19}$. No entanto, as características das provas parecem ser específicas conforme a etapa disputada. Em um dos poucos estudos que avaliaram a importância das diferentes modalidades sobre desempenho total de prova foi observado que em triátlon de longa distância, com distâncias maiores do que o "Ironman", tanto o ciclismo $\left(r^{2}=0,62\right)$ quanto a corrida $\left(\mathrm{r}^{2}=0,87\right)$ foram bem correlacionados com o desempenho total ${ }^{21}$. Esses resultados são parcialmente diferentes dos observados no "Ironman" Brasil em que apenas o ciclismo apresentou boa associação com o desempenho total. Essas diferenças podem ser atribuídas às características da prova, o que sugere a necessidade de se traçar estratégias específicas conforme a etapa a ser disputada. Assim, com base no presente estudo, estratégias para a etapa do "Ironman" Brasil poderiam ser desenvolvidas. No entanto, novos estudos são necessários acerca do treinamento adequado bem como do desenvolvimento de estratégias específicas para o Ironman Brasil.

Com isso, concluímos que não houve evolução significante no tempo total de prova, e no tempo das três modalidades analisadas isoladamente, entre os anos de 2003 a 2010 no "Ironman" Brasil. Entretanto, os resultados podem ter sido influenciados por fatores climáticos ou mudanças no circuito e devem ser utilizados com cautela. Adicionalmente, podemos sugerir que no "Ironman" Brasil o ciclismo é a modalidade determinante para o sucesso do atleta, uma vez que os atletas mais rápidos no ciclismo foram os campeóes da prova em seis dos oito anos analisados. Assim, os nossos resultados podem auxiliar treinadores e atletas na sua preparação e definição da escolha da melhor estratégia utilizada para a disputa do "Ironman" Brasil.

\begin{abstract}
Performance analysis of elite Brazil-Ironman athletes from 2003 to 2010

The aim of this study was to analyze the performance evolution on the Brazil-Ironman from 2003 to 2010. Additionally, we verified which of the modalities were the most influential on the overall performance. The first ten athletes for each year in the ELITE male category were analyzed. The differences between the racing times were analyzed by ANOVA, followed by Tukey post-hoc test. Pearson's correlation coefficient was utilized to investigate the association between races performance and the performance in each one of the different modalities. The results showed that there was no performance evolution over the years and a great variation in racing times was observed. We have found a important correlation between cycling performance and race time $(r=0.82 ; p<0.01)$ and the fastest athlete in cycling was the Brazil-Ironman champion in $75 \%$ of the analyzed years. Thus, it was concluded that there was no evolution in race time over the analyzed years. Additionally, the cycling might be the most influential modality for athletes' success in Brazil-Ironman.
\end{abstract}

KEY WORDS: Athletic performance; Running; Cycling; Swimming.

\title{
Referências
}

1. Lepers R. Analysis of Hawaii ironman performances in elite triathletes from 1981 to 2007. Med Sci Sport Exerc. 2008;40:1828-34.

2. Bentley DJ, Millet GP, Vleck VE, McNaughton LR. Specific aspects of contemporary triathlon: implications for physiological analysis and performance. Sports Med. 2002;32:345-59.

3. O’Toole ML, Douglas PS, Hiller WD. Applied physiology of a triathlon. Sports Med. 1989;8:201-25. 
4. Hawley JA, Hopkins WG. Aerobic glycolytic and aerobic lipolytic power-systems: a new paradigm with implications for endurance and ultraendurance events. Sports Med. 1995;19:240-50.

5. Laursen PB. Long distance triathlon: demands, preparation and performance. J Hum Sport Exerc. 2011;6:247-63.

6. IronmanBrazil. História do Ironman Brazil. Florianópolis: LatinSports; 2009 [citado 2012]; Available from: http:// www.ironmanbrasil.com.br/br/historia.asp.

7. Abbiss CR, Quod MJ, Martin DT, et al. Dynamic pacing strategies during the cycle phase of an Ironman triathlon. Med Sci Sport Exerc. 2006;38:726-34.

8. Bentley DJ, Cox GR, Green D, Laursen PB. Maximising performance in triathlon: applied physiological and nutritional aspects of elite and non-elite competitions. J Sci Med Sport. 2008;11:407-16.

9. Jeukendrup AE, Martin J. Improving cycling performance: how should we spend our time and money. Sports Med. 2001;31:559-69.

10. Faria EW, Parker DL, Faria IE. The science of cycling: factors affecting performance: part 2. Sports Med. 2005;35:313-37.

11. Jeukendrup AE, Jentjens RL, Moseley L. Nutritional considerations in triathlon. Sports Med. 2005;35:163-81.

12. Laursen PB, Suriano R, Quod MJ, et al. Core temperature and hydration status during an Ironman triathlon. Brit J Sport Med. 2006;40:320-5.

13. Laursen PB, Rhodes EC, Langill RH, McKenzie DC, Taunton JE. Relationship of exercise test variables to cycling performance in an Ironman triathlon. Eur J Appl Physiol. 2002;87:433-40.

14. Gulbin JP, Gaffney PT. Ultraendurance triathlon participation: typical race preparation of lower level triathletes. J Sports Med Phys Fitness. 1999;39:12-5.

15. Knechtle B, Wirth A, Alexander Rust C, Rosemann T. The relationship between anthropometry and split performance in recreational male ironman triathletes. Asian J Sports Med. 2011;2:23-30.

16. O’Toole ML. Training for ultraendurance triathlons. Med Sci Sport Exerc. 1989;21:S209-13.

17. Foster C, Lucia A. Running economy: the forgotten factor in elite performance. Sports Med. 2007;37:316-9.

18. DiGeronimo MK. Swimming economy in long distance swimmers and ironman triathletes. Ohio: Ohio State University; 2010.

19. Abbiss CR, Laursen PB. Describing and understanding pacing strategies during athletic competition. Sports Med. 2008;38:239-52.

20. Kimber NE, Ross JJ, Mason SL, Speedy DB. Energy balance during an ironman triathlon in male and female triathletes. Int J Sport Nutr Exerc Metab. 2002;12:47-62.

21. Lepers R, Knechtle B, Knechtle P, Rosemann T. Analysis of ultra-triathlon performances. J Sports Med Phys Fitness. 2011;2:131-6.

\begin{tabular}{r|l} 
ENDEREço & \\
Everton Crivoi Carmo & \\
Escola de Educação Física e Esporte - USP & Recebido para publicação: 21/01/2013 \\
Av. Prof. Mello Moraes, 65 & 1a. Revisão: 16/08/2013 \\
05508-030 - São Paulo - SP - BRASIL & 2a. Revisão: 24/01/2014 \\
e-mail: evertoncrivoi@usp.br & Aceito: 27/01/2014
\end{tabular}

64 • Rev Bras Educ Fís Esporte, (São Paulo) 2014 Jan-Mar; 28(1):57-64 